\title{
Simulation of boron diffusion during low-temperature annealing of implanted silicon
}

\author{
O. I. Velichko and A. P. Kavaliova \\ Department of Physics, Belarusian State University of Informatics \\ and Radioelectronics, 6, P. Brovki Street, Minsk, 220013 Belarus \\ E-mail addresses: velichkomail@gmail.com (Oleg Velichko)
}

\begin{abstract}
Modeling of ion-implanted boron redistribution in silicon crystals during low-temperature annealing with a small thermal budget has been carried out. It was shown that formation of "tails" in the lowconcentration region of impurity profiles occurs due to the long-range migration of boron interstitials.
\end{abstract}

Keywords: Diffusion; Annealing; Doping Effects; Boron; Silicon PACS 66.30.-h, 61.72.Uf, 85.40.Ry

\section{Introduction}

It is well known that device scaling requires decreasing all vertical and lateral dimensions of transistors At present, a low-energy high-dose ion implantation is used for the formation of shallow junctions However, we need to undergo silicon substrate to subsequent annealing to avoid defects created by ion implantation. During thermal treatment of ion implanted layers a great amount of nonequilibrium self-interstitials is generated, which results in transient enhanced diffusion (TED) of impurity atoms. Intensive TED prevents the formation of shallow junctions especially for boron doped regions (see, for example, [1, 2, 3, 4). Therefore, the problem of formation of doped regions with abrupt impurity distributions in vertical and lateral directions requires annealing imposed with an extremely small thermal budget for the TED to be decreased [5]. One of the techniques to provide a small thermal budget and solve this problem is the use of low-temperature annealing of short duration [6]. Another technique widely used to suppress transient enhanced diffusion of ion-implanted impurity is the introduction of boron into 
the silicon layer preamorphized by implantation of Ge ions [7, 8, 9, 10]. As a result of the solid phase epitaxial recrystallization (SPER) of the amorphous layer at the initial stage of annealing a perfect crystalline structure without defects visible by means of electronic microscopy is formed in the array of the implanted boron. Nevertheless, in this case TED is also observed during the subsequent annealing, but of absolutely another character, and characterized by a smaller intensity. Indeed, at low annealing temperatures an extended "tail" in the low-concentration region of boron profile is formed in all the cases mentioned above. This "tail" represents a direct line for logarithmic scale on the concentration axis.

It was shown in [11, 12, 13] that the majority of the cases of "tail" formation in the low-concentration region of ion-implanted impurity distribution, including boron implantation in preamorphized silicon, is related to the phenomenon of a long-range migration of nonequilibrium impurity interstitials, and we can neglect channeling of a fraction of implanted atoms if ion implantation of $\mathrm{In}, \mathrm{P}$, and $\mathrm{Ga}$ is performed in a random direction or B is implanted in a preamorphized layer. In [14, 15], a similar theoretical investigation and simulation of impurity redistribution were carried out for boron implantation in a random direction in the crystalline silicon. It has been shown that the long-range migration of nonequilibrium boron interstitials is responsible for the "tail" formation in a crystal lattice damaged by boron ions implanted at medium fluencies which are insufficient to create an amorphous layer. For example, it was found in [15] that the average migration length of boron interstitials $l_{A I}$ is equal to $24 \mathrm{~nm}$ for annealing at a temperature of $600{ }^{\circ} \mathrm{C}$ for $10 \mathrm{~s}$. This value is two times greater than the average migration length of boron interstitials $l_{A I}=12 \mathrm{~nm}$ and $l_{A I}=11 \mathrm{~nm}$ found for boron diffusion in the preamorphized and recrystallized silicon layer at temperatures of $850{ }^{\circ} \mathrm{C}$ [11] and $800{ }^{\circ} \mathrm{C}$ [16, 17] with annealing duration of $60 \mathrm{~s}$. It is interesting to note that due to the higher temperatures and longer duration a significant increase in the thermal budget occurred in the experiments that were simulated in [11] and [16, 17]. Therefore, it is reasonable to investigate the mechanism of "tail" formation for low-energy boron implantation in crystalline silicon during the subsequent low-temperature annealing with durations longer than $10 \mathrm{~s}$, i. e., for the increased thermal budget. It is worth noting that the elucidation of the mechanisms of "tail" formation has a practical importance because this phenomenon influences the depth of $\boldsymbol{p}$ - $\boldsymbol{n}$ junction, as well as it is actual needed for the investigation of the transport processes of implanted boron atoms. Indeed, it has been shown in [16, 17] 
that the mechanism of boron diffusion varies with a change in the annealing temperature.

\section{Model of boron diffusion}

For simulation of interstitial diffusion of boron atoms, we use the model proposed in [11, 12], which includes the following system of equations:

1) the conservation law for immobile impurity atoms

$$
\frac{\partial C^{T}(x, t)}{\partial t}=\frac{C^{A I}(x, t)}{\tau^{A I}}-G^{A I}(x, t),
$$

2) the diffusion equation for nonequilibrium boron interstitials

$$
d^{A I} \frac{\partial^{2} C^{A I}}{\partial x^{2}}-\frac{C^{A I}}{\tau^{A I}}+G^{A I}(x, t)=0
$$

which can be present in the form suitable for numerical or analytical solution [18,

$$
\frac{\partial^{2} C^{A I}}{\partial x^{2}}-\frac{C^{A I}}{l_{A I}^{2}}+\frac{\tilde{g}^{A I}(x, t)}{l_{A I}^{2}}=0
$$

where

$$
l_{A I}=\sqrt{d^{A I} \tau^{A I}}, \quad \tilde{g}^{A I}(x, t)=G^{A I}(x, t) \tau^{A I} .
$$

Here $C^{T}$ is the total concentration of substitutionally dissolved impurity atoms, impurity atoms incorporated into clusters and trapped by various defects including the extended defects (immobile boron atoms); $C^{A I}$ is the summarized concentration of nonequilibrium boron interstitials in different charge states; $d^{A I}$ and $\tau^{A I}$ are the diffusivity and average lifetime of nonequilibrium impurity interstitials, respectively; $l_{A I}$ and $G^{A I}$ are the average migration length and generation rate of interstitial impurity atoms, respectively. The concentration of impurity interstitials (mobile species) is also included in $C^{T}$.

We use the stationary diffusion equation for interstitial impurity atoms in view of their large average migration length $l_{A I}\left(l_{A I} \gg l_{\text {fall }}\right)$ and due to the small average lifetime of these nonequilibrium interstitials $\tau^{A I}\left(\tau^{A I} \ll \tau_{p r}\right)$. Here $l_{\text {fall }}$ is the characteristic length relevant to the significant decrease of impurity concentration in the high-concentration region and $\tau_{p r}$ is the duration 
of thermal treatment. It is also supposed that the function $G^{A I}(x, t)$ that describes the generation rate of interstitial impurity atoms changes slowly with time, increasing as compared to the value of $\tau^{A I}$.

It is worth noting that according to the experimental data [1, 19], a significant part of boron atoms in the "tail" region is electrically inactive, i.e., impurity atoms do not occupy the substitutional position. A few possible explanations can be given to this phenomenon. For example, the average lifetime of the nonequilibrium boron interstitials $\tau^{A I} \sim \tau_{p r}$, i.e., it is equal to tens of minutes at temperatures of $800{ }^{\circ} \mathrm{C}$ [1] and $900{ }^{\circ} \mathrm{C}$ [19]. However, this assumption contradicts the common point of view that the interstitial impurity atoms are highly mobile Really, $l_{A I}$ is not too large $\left(l_{A I} \sim 20 \mathrm{~nm}\right)$, whereas the duration of annealing is longer than enough and for the high value of diffusivity of boron interstitials a significant value of $l_{A I}$ should be expected. In our opinion, the assumption that the boron interstitials in the "tail" region are being incorporated with the defects of the crystal lattice and, therefore, become immobile is more reasonable. However, this problem requires further investigation.

In contrast to the models of [11, 12], the Pearson type IV distribution [20] is used to describe the spatial distribution of impurity atoms after implantation:

$$
C_{0}(x)=C(x, 0)=C_{m} F^{P}\left(x, R_{p}, \triangle R_{p}, S k\right),
$$

where

$$
C_{m}=\frac{Q}{\sqrt{2 \pi} \Delta R_{p}} \times 10^{-8} \quad\left[\mu m^{-3}\right] .
$$

Here $F^{P}\left(x, R_{p}, \triangle R_{p}, S k\right)$ is the function that describes the Pearson type IV distribution and which is tabulated in [20]; $Q$ is the dose of ion implantation [ion $\left./ \mathrm{cm}^{2}\right] ; R_{p}$ and $\triangle R_{p}$ are the average projective range of implanted ions and straggling of the projective range, respectively; $S k$ is the skewness of the Pearson type IV distribution

On the other hand, to simplify the description for the spatial distribution of generation rate of nonequilibrium boron interstitials we used the Gaussian distribution:

$$
G^{A I}(x, t)=G_{m}^{A I}(t) \exp \left[-\frac{\left(x-R_{m}\right)^{2}}{2 \triangle R_{p}^{2}}\right]
$$


shifted to the position of $R_{m}$ to adjust with the maximum of distribution of implanted ions. Here $R_{m}$ is the position of a maximum of the Pearson type IV distribution for impurity concentration; $G_{m}^{A I}(t)$ is the value of the generation rate for interstitial impurity atoms in the maximum of the Pearson type IV distribution. As well as in the models of [11, 12, it is supposed that boron atoms occur in the interstitial positions either directly during implantation, or during rearrangement or annealing of the clusters that incorporate impurity atoms.

\section{Analytical solution of diffusion equation}

It can be seen from expression (77) that the generation rate of nonequilibrium boron interstitials is approximated by the product of two multipliers, one of them describes the dependence of the generation rate on time, whereas the other describes the spatial distribution of the generation rate of nonequilibrium boron interstitials:

$$
G^{A I}(x, t)=G_{m}^{A I}(t) G_{s p}^{A I}(x),
$$

Let us substitute expression (8) into Eq.(2) and integrate the resulting equation with respect to time over the interval $\left[\mathrm{t}_{0}, \mathrm{t}_{\mathrm{k}}\right]$ :

$$
d^{A I} \frac{d^{2} C_{e f f}^{A I}}{d x^{2}}\left(t_{k}-t_{0}\right)-\frac{C_{e f f}^{A I}}{\tau^{A I}}\left(t_{k}-t_{0}\right)+G_{s p}^{A I}(x) \int_{t_{0}}^{t_{k}} G_{m}^{A I}(t) d t=0
$$

or

$$
d^{A I} \frac{d^{2} C_{e f f}^{A I}}{d x^{2}}-\frac{C_{e f f}^{A I}}{\tau^{A I}}+g_{m k}^{A I} G_{s p}^{A I}(x)=0
$$

where

$$
\begin{aligned}
C_{e f f}^{A I}(x) & =\frac{1}{\left(t_{k}-t_{0}\right)} \int_{t_{0}}^{t_{k}} C^{A I}(x, t) d t \\
g_{m k}^{A I} & =\frac{1}{\left(t_{k}-t_{0}\right)} \int_{t_{0}}^{t_{k}} G_{m}^{A I}(t) d t
\end{aligned}
$$


Here $C_{e f f}^{A I}(x)$ and $g_{m k}^{A I}$ are, respectively, the concentration of impurity interstitials and the value of the generation rate for boron interstitials in the maximum of space distribution which are time-averaged on the interval $\left[\mathrm{t}_{0}, \mathrm{t}_{\mathrm{k}}\right]$.

It follows from the transformations performed that for the case of the generation rate for boron interstitials, representing the product of time- and space dependent multipliers, the system of Eqs.(11) and (2) is similar to the system of equations (11) and (10) with the constant coefficient $g_{m k}^{A I}$. It is worth noting that Eq.(10) can be solved analytically. For example, such solution was obtained in [18] for

$$
g_{m k}^{A I} G_{s p}^{A I}(x)=g_{m k}^{A I} \exp \left[-\frac{\left(x-R_{m}\right)^{2}}{2 \Delta R_{p}^{2}}\right] .
$$

It is well known that the intensity of transient enhanced diffusion decreases with time. Let us suppose that

$$
G_{m}^{A I}(t)=G_{m 0}^{A I} \exp \left(-\frac{t-t_{0}}{\tau_{g e n}}\right)
$$

where $\tau_{\text {gen }}$ is the relaxation time for the generation of nonequilibrium impurity interstitials and $G_{m 0}^{A I}$ is the value of the generation rate in the maximum of the Pearson type IV distribution for $\mathrm{t}=\mathrm{t}_{0}$.

The parameters $G_{m 0}^{A I}$ and $\tau_{g e n}$ can be derived if we obtain two values of $g_{m k}^{A I}$ for two thermal treatments with different durations. Indeed, let us suppose for simplicity that $t_{0}=0$ and $g_{m 1}^{A I}$ and $g_{m 2}^{A I}$ are the values of the generation rate for boron interstitials in the maximum of space distribution time-averaged on the intervals $\left[0, \mathrm{t}_{1}\right]$ and $\left[0, \mathrm{t}_{2}\right]$, respectively. Then, the following system of two independent algebraic equations is valid:

$$
\begin{aligned}
& g_{m 1}^{A I}=G_{m 0}^{A I} \tau_{g e n}\left[1-\exp \left(-\frac{t_{1}}{\tau_{g e n}}\right)\right], \\
& g_{m 2}^{A I}=G_{m 0}^{A I} \tau_{g e n}\left[1-\exp \left(-\frac{t_{2}}{\tau_{\text {gen }}}\right)\right] .
\end{aligned}
$$

To obtain the solution of the system (15), (16), let us specify the quantity A as $A=g_{m 1}^{A I} t_{1} /\left(g_{m 2}^{A I} t_{2}\right)$. Then, the value of $\tau_{g e n}$ can be obtained by solving the nonlinear equation: 


$$
\exp \left(-\frac{t_{1}}{\tau_{\text {gen }}}\right)-A \exp \left(-\frac{t_{2}}{\tau_{g e n}}\right)+(A-1)=0 .
$$

Müller's iteration method of successive bisections and inverse parabolic interpolation 21 was used in this work to solve Eq.(17). Then, we obtained the value of $G_{m 0}^{A I}$ from Eq.(15) or Eq.(16).

\section{Simulation results}

The results of simulation of boron interstitial diffusion within the framework of the model described above are presented in Figs. 1] and 2. For comparison we use the experimental data of [6]. In paper [6], implantation of boron ions was carried out with a dose of $1 \times 10^{14} \mathrm{~cm}^{-2}$ and an energy of $1 \mathrm{keV}$ in silicon substrates of orientation (100) under $7^{\circ}$ tilt, $30^{\circ}$ twist angles to prevent channeling. Before implantation a pure silicon layer $(10-\mu \mathrm{m}$ thick, $\boldsymbol{p}$-type conductivity with a resistivity of $10 \Omega \mathrm{m}$ ) has been grown up epitaxially. The chemical profiles of the samples before and after annealing were measured by means of the high depth resolution secondary ion mass spectrometry (SIMS). The annealing of the samples was carried out at a temperature of $600{ }^{\circ} \mathrm{C}$ for 10,600 , and $1200 \mathrm{~s}$.

It follows from the experimental data of [6] that the boron concentration profile after implantation is characterized by a significant asymmetry. For this reason, the Pearson type IV distribution was used to calculate the concentration profile of ion-implanted boron. It was found in [15] that the values of $R_{p}=0.007 \mu \mathrm{m}, \triangle R_{p}=0.0055 \mu \mathrm{m}$, and $S k=1.2$ give the best fit to the experimental data of [6]. It is necessary to note that the Pearson type IV distribution with obtained values of the parameters gives a good fit for the whole impurity concentration profile excluding the low-concentration region. The problem of the accurate calculation of boron concentration profile in the low-concentration region has been also considered in [15] where it was supposed that heating of silicon substrate takes place during the ion implantation described in [6]. Then, due to the elevated temperature, a fast interstitial diffusion of boron atoms that occupy interstitial position occurs which results in the formation of the "tail" in the low-concentration region of boron profile. It was found in [15] that the following values of the parameters describing the process of interstitial diffusion allow us to obtain a good fit to the boron profile in the low-concentration region, namely: the 


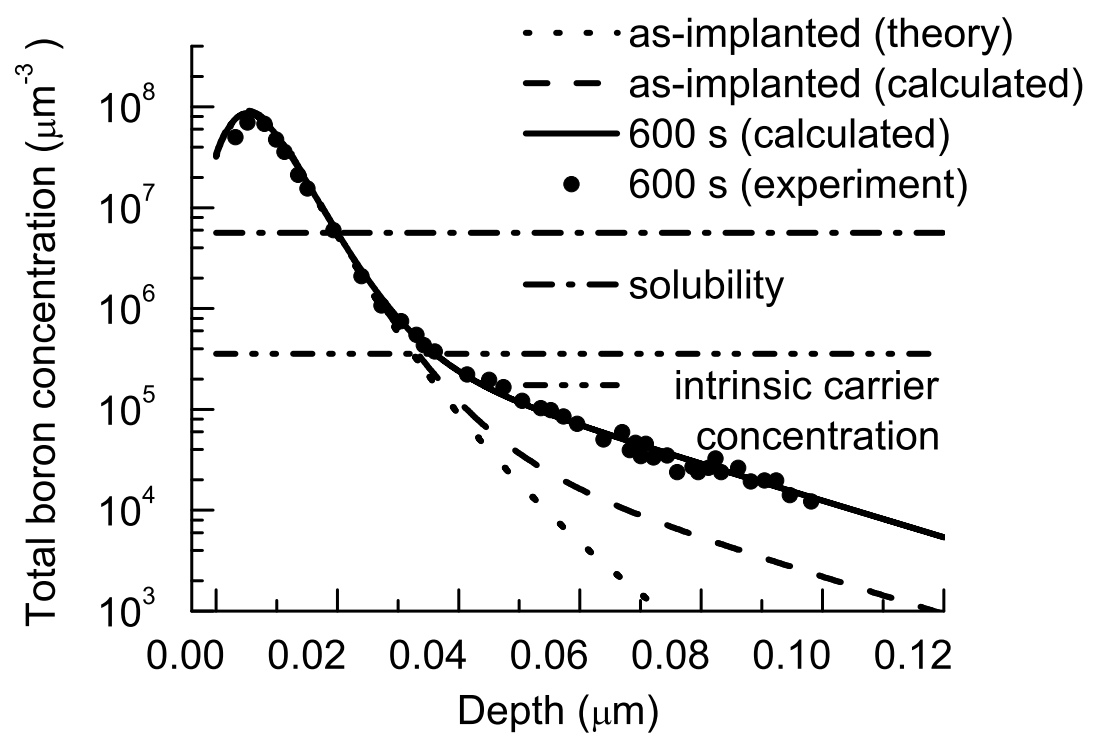

Figure 1: Calculated boron concentration profile after annealing for $600 \mathrm{~s}$ at a temperature of $600{ }^{\circ} \mathrm{C}$. The dotted curve represents boron concentration profile after ion implantation at an energy of $1 \mathrm{keV}$ with a dose of $1 \times 10^{14}$ $\mathrm{cm}^{-2}$ described by the Pearson type IV distribution. The dashed curve represents boron concentration profile calculated under the assumption of the long-range interstitial migration of a part of the impurity atoms during ion implantation to provide fitting with the experimental data for boron distribution after implantation [6]. The experimental data for boron concentration after annealing (filled circles) are also taken from [6]

average migration length of boron interstitials $l_{A I}=0.024 \mu \mathrm{m}(24 \mathrm{~nm})$ and a part of boron atoms participating in the interstitial diffusion during implantation $p_{A I}$ is approximately equal to $0.3 \%$. In addition, it was shown in [15] that during subsequent annealing at a temperature of $600{ }^{\circ} \mathrm{C}$ for 10 $\mathrm{s}$ the interstitial boron diffusion is characterized by the same value of the average migration length of impurity interstitials $l_{A I}=24 \mathrm{~nm}$. The best fit to the boron concentration profile after annealing was obtained under the assumption that approximately $0.7 \%$ of the implanted boron atoms entering into the interstitial position in the high-concentration region of ion-implanted 


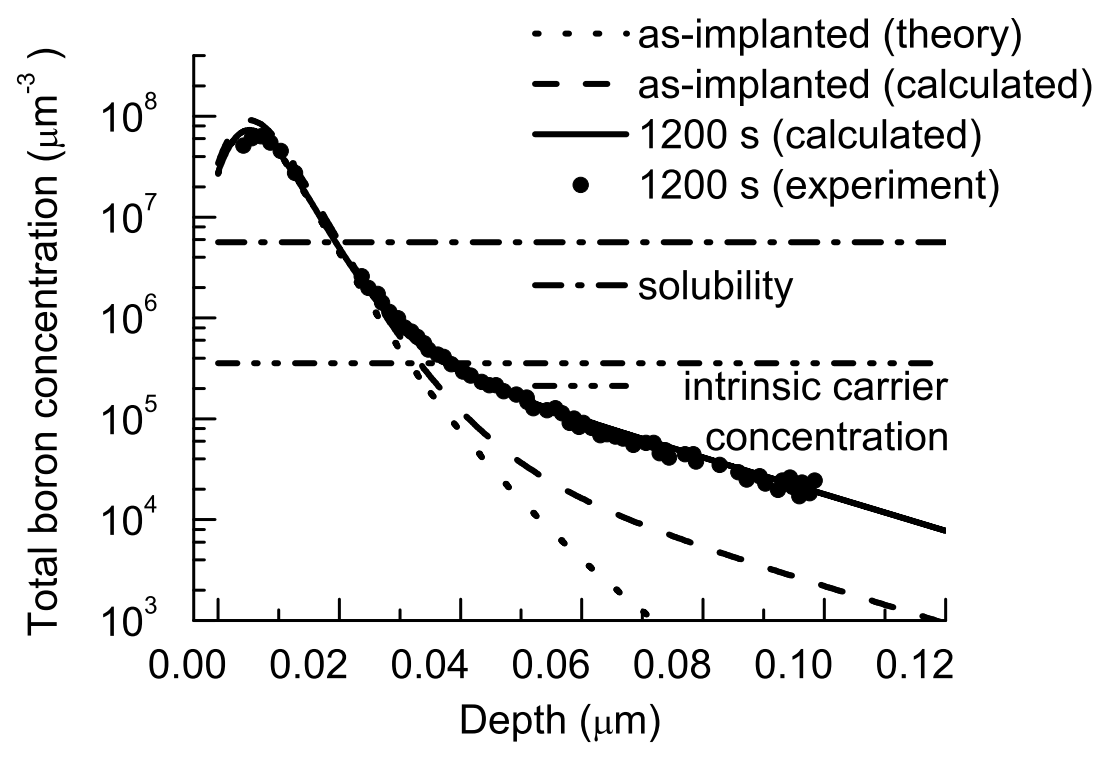

Figure 2: Calculated boron concentration profile after annealing for $600 \mathrm{~s}$ at a temperature of $600{ }^{\circ} \mathrm{C}$. The dotted curve represents boron concentration profile after ion implantation at an energy of $1 \mathrm{keV}$ with a dose of $1 \times 10^{14}$ $\mathrm{cm}^{-2}$ described by the Pearson type IV distribution. The dashed curve represents boron concentration profile calculated under the assumption of the long-range interstitial migration of a part of the impurity atoms during ion implantation. The experimental data for boron concentration after annealing (filled circles) are taken from [6]

impurity participated in the fast interstitial diffusion during annealing and then became immobile again, occupying substitutional positions or forming complexes with the crystal lattice defects.

Taking into account the results of [15], in this work we used the values of $R_{p}=0.007 \mu \mathrm{m}, \triangle R_{p}=0.0055 \mu \mathrm{m}, S k=1.2, l_{A I}=0.024 \mu \mathrm{m}$, and $p_{A I}=$ $0.308 \%$ to calculate the boron concentration profile after implantation. For this value of skewness $S k, R_{m}=0.00521 \mu \mathrm{m}$. As can be seen from Figs. 1 and 2, the results of simulation of boron diffusion during annealing for 600 and $1200 \mathrm{~s}$ agree well with the experimental data if the same value of the average migration length of impurity interstitials $l_{A I}=24 \mathrm{~nm}$ is used. Taking into 
account the results of [15] and the present calculations, one can conclude that the migration length of impurity interstitials varies negligibly with increase of annealing duration. A good fit to the experimental data obtained for different annealing durations with the same value of $l_{A I}$ confirms the correctness of the developed model of the long-range migration for boron interstitial atoms in ion-implanted layers during low-temperature annealing.

\section{Discussion}

It is worth noting that in paper [22] the error arising due to the use of Gaussian distribution instead of the Pearson type IV distribution when modeling the redistribution of ion-implanted impurity was investigated. It was supposed that the Fick's law describes the diffusion of impurity atoms. As follows from the results of [22], the error in calculating the impurity concentration profile is less than $5 \%$ if $L>5\left|R_{m}-R_{p}\right|$. Here $L=\sqrt{D \tau_{p r} C^{A I} / C_{i}^{A I}}$ is the average length of the boron redistribution due to the pair diffusion mechanism; $D$ is the diffusivity of impurity atoms; $C^{A I}$ is the concentration of nonequilibrium silicon self-interstitials; $C_{i}^{A I}$ is the thermally equilibrium value of self-interstitials. Extrapolating the results of [22] to the case of diffusion of impurity interstitials and taking into account that for the experimental data of [6] simulated in this paper $\left|R_{m}-R_{p}\right|$ is equal to $1.79 \mathrm{~nm}$ whereas $l_{A I}=24 \mathrm{~nm}$, we can conclude that using the Gaussian distribution to describe the generation rate of boron interstitials is quite reasonable. In addition, in our computations the Gaussian distribution of generation rate is shifted to the position of $R_{m}$ and, therefore, the error in calculating the concentration profile for nonequilibrium boron interstitials is quite negligible.

The best fit to the boron concentration profiles after annealing presented in Figs. 1 and 2 was obtained under the assumption that approximately 1.5 $\%$ and $2.29 \%$ of the implanted boron atoms participated temporally in the fast interstitial diffusion during annealing for 600 and $1200 \mathrm{~s}$, respectively. It follows from these values and the value of $p_{A I}=0.7 \%$ for the part of boron atoms participating in interstitial diffusion during annealing for $10 \mathrm{~s}$ [15] that there is a decrease in the generation rate of boron interstitials with increase in annealing duration. Indeed, the time-average interstitial generation rates for the intervals $[0,10] \mathrm{s},[0,600] \mathrm{s}$, and $[0,1200] \mathrm{s}$ are $g_{m 10}^{A I}=6.3 \times 10^{5}, g_{m 600}^{A I}$ $=2.19 \times 10^{3}$, and $g_{m 1200}^{A I}=1.675 \times 10^{3} \mu \mathrm{m}^{-3} \mathrm{~s}^{-1}$, respectively. The solution of the system (15), (16) for the time intervals $[0,10] \mathrm{s}$ and $[0,600] \mathrm{s}$ gives the 
following values of the parameters of time-dependent function (14) for the generation rate of boron interstitials: $G_{m 0}^{A I}=8.5655 \times 10^{4} \mu \mathrm{m}^{-3} \mathrm{~s}^{-1}$ and $\tau_{\text {gen }}=$ $15.33 \mathrm{~s}$. The kinetics of generation rate for nonequilibrium boron interstitials calculated for these values of $G_{m 0}^{A I}$ and $\tau_{g e n}$ is presented in Fig. 3. A similar calculation for the intervals [0-10] s and [0-1200] s gives the following values of the kinetics parameters: $G_{m 0}^{A I}=7.5473 \times 10^{4} \mu \mathrm{m}^{-3} \mathrm{~s}^{-1}$ and $\tau_{g e n}=26.6 \mathrm{~s}$. It follows from the values obtained that the main fraction of boron interstitials is generated during annealing duration shorter than $30 \mathrm{~s}$. Therefore, the additional experiments with annealing durations in the range of $10-600 \mathrm{~s}$ are needed to improve the accuracy of kinetics determination.

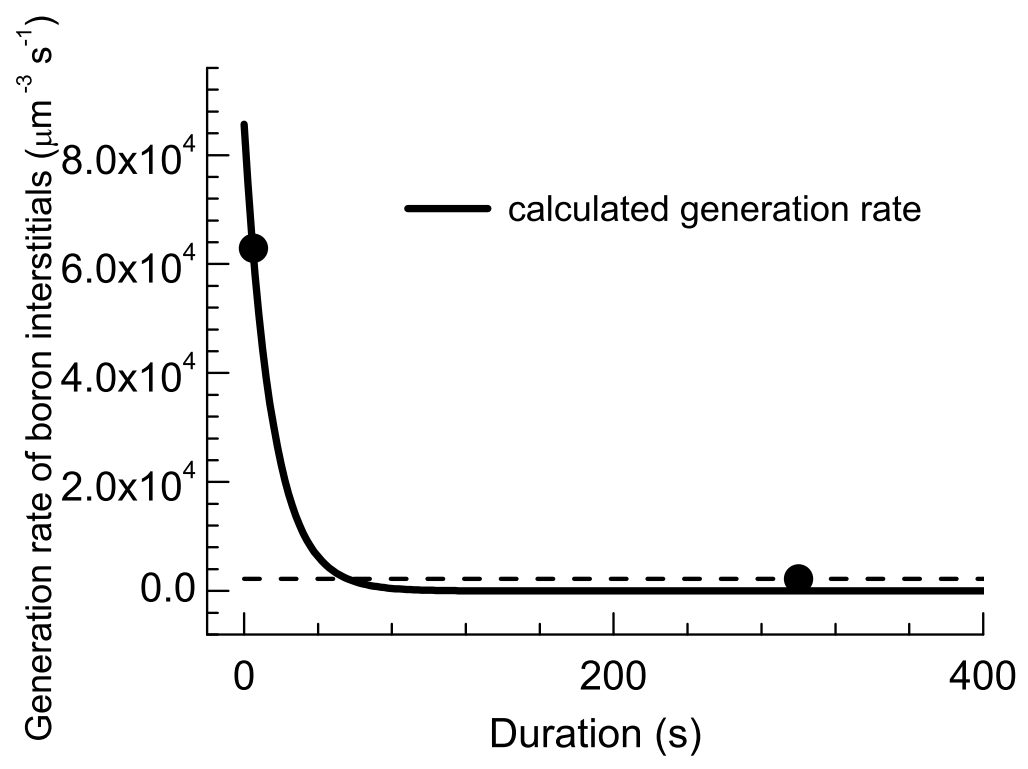

Figure 3: Calculated kinetics of the generation rate of interstitial boron atoms during annealing at a temperature of $600{ }^{\circ} \mathrm{C}$. The filled circle represents the time-average generation rate of boron interstitials for the interval from 0 $\mathrm{s}$ to $10 \mathrm{~s}$. The dashed curve with filled circle represents the time-average generation rate of boron interstitials for the interval from $0 \mathrm{~s}$ to $600 \mathrm{~s}$

It is worth noting that for simulation of boron redistribution during annealing of ion-implanted layers the mechanism of transient enhanced diffusion 
due to formation, migration and dissociation of the "boron atom - silicon selfinterstitial" pairs is usually used. However, we can neglect this mechanism of boron diffusion in simulating the experimental data of [6] due to the very low temperature of annealing and, hence, due to the very small thermal budget. Indeed, the boron diffusivity $D$ for a temperature of $600{ }^{\circ} \mathrm{C}$ calculated according to the results of [23] is equal to $1.78 \times 10^{-13} \mu \mathrm{m}^{2} \mathrm{~s}^{-1}$. Then the average length of boron redistributions due to the pair diffusion mechanism $L=\sqrt{D \tau_{p r} C^{A I} / C_{i}^{A I}}$ equals $1.4 \times 10^{-3} \mu \mathrm{m}(1.4 \mathrm{~nm})$ for annealing duration of $1200 \mathrm{~s}$ and for concentration of nonequilibrium silicon self-interstitials $C^{A I}$ is 10000 times higher than the thermally equilibrium value of this species $C_{i}^{A I}$. Therefore, it is possible to neglect the traditional transient enhanced diffusion Really, one can see from Figs. 1 and 2 that there is no boron redistribution in the high-concentration region of impurity.

It is interesting to compare the derived value $l_{A I}=24 \mathrm{~nm}$ for B implanted in crystalline silicon with the values of the average migration length of boron interstitials obtained for the case of $\mathrm{B}$ implantation in the layer of silicon preamorphized by Ge ions. For example, in [8] $<100>$ oriented, Czochralski grown silicon substrates of the $\boldsymbol{n}$-type of conductivity and resistivity of about 10-25 $\Omega \mathrm{m}$, were used for such experiments. These substrates were implanted with Ge ions at $32 \mathrm{keV}$ to a dose of $1 \times 10^{15} \mathrm{~cm}^{-2}$ that provides amorphization to a depth of $\sim 55 \mathrm{~nm}$, as determined by Rutherford backscattering spectrometry (RBS) and cross-sectional transmission electron microscopy (XTEM). Boron was subsequently implanted at $500 \mathrm{eV}$ to a dose of $2 \times 10^{15} \mathrm{~cm}^{-2}$ at ${ }^{\circ}$ tilt and ${ }^{\circ}$ twist. A $1.5 \mathrm{MeV}$ He beam at $45^{\circ}$ to the sample were used for RBS. After implantation, samples received isochronal rapid thermal annealing in the $\mathrm{N}_{2}$ ambient at a temperature of $850{ }^{\circ} \mathrm{C}$ for $60 \mathrm{~s}$. Atomic profiles of $\mathrm{B}$ were measured by SIMS. In [11] modeling of the doping process described above was carried out, and the average length of boron interstitials $l_{A I}=12$ nm was obtained. In [9] boron implantation with an energy of $1 \mathrm{keV}$ and a dose of $1.5 \times 10^{15} \mathrm{~cm}^{-2}$ was carried out in (100) oriented silicon substrates with the $\boldsymbol{n}$-type of conductivity. For suppression of the transient enhanced diffusion a near surface layer of silicon was preamorphized by implantation of Ge ions with an energy of $15 \mathrm{eV}$. After boron implantation, the thermal annealing in the nitrogen ambient was carried out for $60 \mathrm{~s}$ at a temperature of $800{ }^{\circ} \mathrm{C}$. Calculation of the redistribution of ion-implanted boron for this experimental environment performed in [13] has shown a good agreement with the experimental data for the average migration length of boron interstitials $l_{A I}=11 \mathrm{~nm}$. 
It is clear from the presented results that the average migration length of interstitial boron atoms in crystalline silicon is significantly larger than in the silicon preamorphized by Ge ions and recrystallized due to the solid phase epitaxy at the initial stage of annealing. The obtained result confirms the strong interaction of migrating boron interstitials with Ge atoms previously implanted in the silicon layer. This interaction provides approximately a quadruple reduction of the average lifetime of boron interstitials and the relevant decrease in their migration length.

\section{Conclusions}

Simulation of impurity redistribution for boron implanted in crystalline silicon during annealing at a temperature of $600{ }^{\circ} \mathrm{C}$ for 600 and $1200 \mathrm{~s}$ has been carried out. The results obtained extend and deepen the investigation described in [15] where boron diffusion for 10-s annealing has been simulated. The boron concentration profiles calculated within the framework of the model of the long-range migration of impurity interstitials agree well with the experimental data for low-temperature thermal treatment of crystalline silicon. It is interesting to note that the same value of the average migration length of nonequilibrium boron interstitials $l_{A I}=24 \mathrm{~nm}$ can be used to explain, within the experimental error, the formation of extended "tails" in the low-concentration region of boron concentration profile for annealing duration of 10,600, and $1200 \mathrm{~s}$ as well as for explaining the fast interstitial diffusion during ion implantation It was also shown that approximately 1.5 $\%$ and $2.29 \%$ of the implanted atoms occupied temporally the interstitial position during annealing for 600 and $1200 \mathrm{~s}$, respectively, participated in the fast diffusion, and then became immobile again, took up the substitutional position or formed complexes with defects of the crystalline lattice. It follows from the obtained values of the relaxation time for generation of nonequilibrium impurity interstitials that the main fraction of boron interstitials is generated during annealing duration shorter than $30 \mathrm{~s}$.

A comparison of the present calculations with the earlier published data shows that the obtained value of $l_{A I}$ is approximately two times greater than the average migration length of boron interstitials in the silicon layers preamorphized due to Ge ion implantation and recrystallized by means of solid phase epitaxy at the initial stage of annealing. It means that the migrating boron interstitials interact intensively with Ge atoms, which have 
been used for the formation of a near-surface amorphous layer before boron implantation.

\section{References}

[1] P. A. Stolk, H.-J. Gossmann, D. J. Eaglesham, D. C. Jacobson, C. S.Rafferty, G. H. Gilmer, M. Jaraiz, J. M. Poate, H. S. Luftman, T. E. Haynes, Physical mechanisms of transient enhanced dopant diffusion in ion-implanted silicon, J. Appl. Phys. 1997. Vol.81, No.9. pp.60316050 .

[2] S. C. Jain, W. Schoenmaker, R. Lindsay, P. A. Stolk, S. Decoutere, M. Willander, H. E. Maes, Transient enhanced diffusion of boron in Si, J. Appl. Phys. 2002. Vol.91, No.11. pp.8919-8940.

[3] L. Shao, J. Liu, Q. Y. Chen, Wei-Kan Chu, Boron diffusion in silicon: the anomalies and control by point defect engineering, Mat. Sci. Eng. R. 2003. Vol.42. pp. 65-114.

[4] T. Philippe, S. Duguay, D.Mathiot, D. Blavette, Atomic scale evidence of the suppression of boron clustering in implanted silicon by carbon coimplantation, J. Appl. Phys. 2011. Vol.109. Art. No.023501.

[5] http://www.itrs.net/Links/2011ITRS/Home2011.htm International Technology Roadmap for Semiconductors. 2011 Edition. Front End Processes. p.36.

[6] E. Napolitani, A. Carnera, E. Schroer, V. Privitera, F. Priolo, S Moffatt, Microscopical aspects of boron diffusion in ultralow energy implanted silicon, Appl. Phys. Lett. 1999. Vol.75, No.13. pp.1869-1871.

[7] F. Cristiano, N. Cherkashin, P. Calvo, Y. Lamrani, X. Hebras, A. Claverie, W. Lerch, S. Paul, Thermal stability of boron electrical activation in preamorphized ultra-shallow junctions, Mater. Sci. Eng. B. 2004. Vols.114-155. pp.174-179.

[8] J. J. Hamilton, K. J. Kirkby, N. E. B. Cowern, E. J. H. Collart, M. Bersani, D. Giubertoni, S. Gennaro, A Parisini, Boron deactivation in preamorphized silicon on insulator: Efficiency of the buried oxide as an interstitial sink, Appl. Phys. Lett. 2007. Vol.91, Art. No.092122. 
[9] S. H. Yeong, B. Colombeau, K. R. C Mok, The impact of nitrogen co-implantation on boron ultra-shallow junction formation and underlying physical understanding, Mat. Sci. Eng. B. 2008. Vols.154-155. pp.43-48.

[10] D. De Salvador, E. Napolitani, G. Bisognin, M. Pesce, A. Carnera, E. Bruno, G. Impellizzeri, S. Mirabella, Boron diffusion in extrinsically doped crystalline silicon, Phys. Rev. B. 2010. Vol.81, Art. No.045209.

[11] O. I. Velichko, N. V. Kniazhava, Modeling of the long-range interstitial migration of ion implanted boron, Comput. Mat. Sci. 2010. Vol.48. pp.409-412.

[12] O. I. Velichko, A. P. Kavaliova, Simulation of ion-implanted boron redistribution under different conditions of the transient enhanced diffusion suppression, in: V.M. Anischik et al. (Eds.) Proceedings of the 9-th International Conference "Interaction of Radiation with Solids": September 20-22 2011, Publication Center of BSU, Minsk, Belarus 2011. pp.115-117 (In Russian).

[13] O. I. Velichko, A. P. Kavaliova, Modeling of the transient interstitial diffusion of implanted atoms during low-temperature annealing of silicon substrates, Physica B. 2012. Vol.407. pp.2176-2184.

[14] N. V. Kniazhava, Modeling of the interstitial boron diffusion in nanometer layers formed by low energy ion implantation, in: E.A. Rovba et al. (Eds.) Proc. of the XVIII Republican Scientific Conf. "Fizika kondensirovannogo sostoyaniya (Physics of condensed state)", Grodno, April 21-23, 2010. - GrSU, Grodno, Belarus, 2010. pp.46-48.

[15] O. I. Velichko, V.V. Axenov, A. P. Kavaliova, Modeling of boron interstitial diffusion in crystalline silicon, Inzhenerno-Fizicheskii Zhurnal. 2012. Vol. 85, No.4, pp.851-855 (In Russian).

[16] O. I. Velichko, A. A. Hundorina, Simulation of boron redistribution process during annealing of silicon layers undergone a high fluence ion implantation, in: V.B. Odzhaev et al. (Eds.) Proc. of IV Int. Scientific Conf. "Materials and Structures of Modern Electronics", Minsk, September 23-24, 2010. - Publication Center of BSU, Minsk, Belarus, 2010. pp.112-115. 
[17] O. I. Velichko, A. A. Hundorina, Change in the diffusion mechanisms of boron implanted into silicon with increase in the annealing temperature, Nonlinear Phenom. Complex Syst. Vol.14, No.4. (2011) pp.384390.

[18] O. I. Velichko, N.A. Sobolevskaya, Analytical solution of the equations describing interstitial migration of impurity atoms, Nonlinear Phenom. Complex Syst. 2011. Vol.14, No.1. pp.70-79.

[19] W. K. Hofker, Implantation of boron in silicon, Philips Res. Repts. Suppl. 1975. No.8. pp.1-121.

[20] A. F. Burenkov, F. F. Komarov, M. A. Kumakhov, M. M. Temkin, Prostranstvennye Raspredeleniya Energii, Vydelennoi v Kaskade Atomnyh Stolknovenii v Tverdykh Telakh (Space Distributions of Energy Deposited in Atomic Collisions in Solids), Energoatomizdat, Moscow, 1985 (in Russian).

[21] G. K. Kristiansen, Zero of arbitrary function, BIT. 1963. Vol.3. pp.205-206.

[22] A. F. Burenkov, O. I. Velichko, V. D. Kuryazov, A. A. Labuda, V. K. Malevko, E. E. Tkharev, Characteristics of calculations of diffusion profiles for impurity distribution after implantation of high energy ions, in: Fizika poluprovodnikov i mikroelektronika (Semiconductor physics and microelectronics), Volume of scientific papers of the Institutes of Higher Education, Eds. (P. T. Oreshkin, A. N. Gubkin, G. A. Kurov, V. F. Sinorov, A. I. Pereligin, T. A. Kholomina).Ryazan State Radio Engineering Institute, Ryazan, 1980. pp.119-122 (In Russian).

[23] Y. M. Haddara, B. T. Folmer, M. E. Law, T. Buyuklimanli, Accurate measurements of the intrinsic diffusivities of boron and phosphorus in silicon, Appl. Phys. Lett. 2000. Vol.77, No.13. pp.1976-1978. 